\title{
A re-examination of twitching motility in Pseudomonas aeruginosa
}

\author{
Annalese B. T. Semmler, ${ }^{1,2}$ Cynthia B. Whitchurch ${ }^{1}$ and John S. Mattick ${ }^{1,2}$
}

Author for correspondence: John S. Mattick. Tel: +6173365 4446. Fax: +61733654388. e-mail: j.mattick@cmcb.uq.edu.au

Centre for Molecular and Cellular Biology ${ }^{1}$ and Department of Biochemistry2, University of Queensland, Brisbane, QLD 4072, Australia

\begin{abstract}
Twitching motility is a form of solid surface translocation which occurs in a wide range of bacteria and which is dependent on the presence of functional type IV fimbriae or pili. A detailed examination of twitching motility in Pseudomonas aeruginosa under optimal conditions in vitro was carried out. Under these conditions (at the smooth surface formed between semi-solid growth media and plastic or glass surfaces) twitching motility is extremely rapid, leading to an overall radial rate of colony expansion of $0.6 \mathrm{~mm} \mathrm{~h}^{-1}$ or greater. The zones of colony expansion due to twitching motility are very thin and are best visualized by staining. These zones exhibit concentric rings in which there is a high density of microcolonies, which may reflect periods of expansion and consolidation/cell division. Video microscopic analysis showed that twitching motility involves the initial formation of large projections or rafts of aggregated cells which move away from the colony edge. Behind the rafts, individual cells move rapidly up and down trails which thin and branch out, ultimately forming a fine lattice-like network of cells. The bacteria in the lattice network then appear to settle and divide to fill out the colonized space. Our observations redefine twitching motility as a rapid, highly organized mechanism of bacterial translocation by which $P$. aeruginosa can disperse itself over large areas to colonize new territories. It is also now clear, both morphologically and genetically, that twitching motility and social gliding motility, such as occurs in Myxococcus xanthus, are essentially the same process.
\end{abstract}

Keywords: type IV fimbriae, pili, surface translocation, gliding motility

\section{INTRODUCTION}

The term twitching motility was first coined by Lautrop (1961) to describe flagellar-independent surface motility in Acinetobacter calcoaceticus. Similar motility has been subsequently described in a wide range of other bacteria, including Pseudomonas aeruginosa, Neisseria gonorrhoeae, Neisseria meningitidis and other Neisseriaceae, various Moraxella species, Dichelobacter nodosus, Branhamella catarrhalis, Suttonella indologenes, Alteromonas putrefaciens, Pasteurella multocida, Xanthomonas maltophila, Kingella denitrificans and many others (Mattick et al., 1993).

Henrichsen (1972) defined twitching motility as a kind of surface translocation that produced spreading zones

Abbreviations: Tc, tetracycline; TTC, 2,3,5-tetraphenyltetrazolium chloride or tetrazolium red. on solid media, in which the colony edge exhibited varying micromorphological patterns, with cell movement being 'intermittent and jerky' and 'predominantly singly, although smaller moving aggregates occur'. In some cases, such as N. gonorrhoeae and N. meningitidis, the twitching zones on agar are so small as to be almost invisible (Henrichsen, 1975), suggesting that the activity of twitching motility is variable in different species and/or that the culture conditions in vitro are suboptimal for this process. He also concluded that twitching motility on agar is dependent on a surface film of liquid (and accompanying humidity), as well as the presence of polar fimbriae, later termed type IV fimbriae (or pili) (Henrichsen \& Blom, 1975; Henrichsen, 1983; Ottow, 1975; Mattick et al., 1987).

Bradley carried out detailed microscopic studies of wildtype $P$. aeruginosa in comparison to non-fimbriated and hyperfimbriated mutants which lack twitching motility. He confirmed that fimbriae are required for twitching 
motility and concluded that the mechanical basis of this process is fimbrial extension and retraction (Bradley, 1980), a hypothesis which has been broadly accepted in the absence of further information. Type IV fimbriae are filaments of about $6 \mathrm{~nm}$ in diameter which range up to several micrometres in length. They occur at one or both poles of the bacterium and are primarily composed of a small structural subunit (pilin or PilA in $P$. aeruginosa) with a characteristic highly conserved and highly hydrophobic amino-terminal region, which forms the core of the helical structure, whose outer face is comprised of the more hydrophilic and more variable domains of the subunit (Dalrymple \& Mattick, 1987; Parge et al., 1995; Forest \& Tainer, 1997). The superficial similarity of these structures to the peritrichous type I fimbriae found in Escherichia coli led many investigators to assume that the (primary) function of these organelles is attachment. There is substantial experimental evidence for adhesive properties of type IV fimbriae and reduced cellular adhesion in their absence, both in P. aeruginosa and other species (Farinha et al., 1994; Rothbard et al., 1985; Ruehl et al., 1993; Chi et al., 1991). By and large, however, the role(s) of type IV fimbriae in adhesion and twitching motility have been studied independently.

In recent years $P$. aeruginosa has been used as the primary model for studying fimbrial biology, due to its ease of culture and advanced genetics. Characterization of $P$. aeruginosa mutants which lack twitching motility has so far identified over 30 genes involved in fimbrial biogenesis and function, many of which have homology to other gene/protein sets involved in protein secretion and DNA uptake in various bacteria (Hobbs \& Mattick, 1993; Alm \& Mattick, 1997). This includes multiple subunits with the characteristic type IV amino-terminal sequence, a specific prepeptidase, nucleotide-binding proteins and inner- and outer-membrane proteins. It is now evident that all of these systems share a common assembly pathway and architecture, as well as in all likelihood a common mechanism of action, based on rotation of a helical structure formed by subunits which share a common core but which have different external domains adapted to different functions. It has also been shown that fimbrial production and twitching motility in $P$. aeruginosa are controlled by at least three signal transduction systems, one of which is analogous to, but significantly more complex than, the chemotactic transmitter-receiver system controlling flagellar rotation in E. coli (Hobbs et al., 1993; Darzins, 1993, 1994, 1995; Whitchurch et al., 1996; Alm \& Mattick, 1997).

Related genes encoding the type IV fimbrial subunit and other components have been found in a large number of bacteria known to exhibit twitching motility, as well as in some not previously recognized to possess type IV fimbriae, including Myxococcus xanthus (Wu \& Kaiser, 1995), Aeromonas spp. (Barnett et al., 1997), Legionella pneumophila (Liles et al., 1998; Stone \& Abu Kwaik, 1998), Pseudomonas syringae (Roine et al., 1998) and Azoarcus spp. (Dorr et al., 1998), the latter indicating that type IV fimbriae are important in bacterial coloniz- ation not only of animals but also of plants, fungi and protozoa.

Despite considerable knowledge of the molecular genetics of type IV fimbriae, twitching motility as a process per se has not been carefully examined since the studies of Henrichsen and Bradley some 15-25 years ago. On the surface of agar plates twitching zones are small, presenting as narrow 'ground-glass' type edges around colonies which also appear rough (Fig. 1a). However, in recent years it has become clear that some species, notably Moraxella bovis (McMichael, 1992) and P. aeruginosa (Darzins, 1993; Alm \& Mattick, 1995), exhibit very active twitching motility at the interstitial surface between agar and plastic or glass, leading to spreading zones approaching $2 \mathrm{~cm}$ in diameter after overnight growth (Fig. 1c). Here we have used video microscopic techniques to re-examine this process in $P$. aeruginosa under culture conditions which promote very active twitching motility. The results show that twitching motility is a highly organized process which permits rapid colonization of surfaces. Our observations also indicate that twitching motility and the social component of gliding motility (as occurs in the myxobacteria) are equivalent.

\section{METHODS}

Bacterial strains and media. The $P$. aeruginosa strains used in this study were PAK (D. Bradley, Memorial University of Newfoundland, St John's, Canada) and PAKpilA:: Tc ${ }^{\mathrm{R}}$ (formally strain AWK described by Watson et al., 1996b). P. aeruginosa was maintained on LB medium (Sambrook et al., 1989) solidified with $1.5 \%$ agar. Subsurface twitching assays were performed using LB medium solidified with $1 \%$ agar. Light microscopy was performed using nutrient medium (g $\mathrm{l}^{-1}$ : tryptone, 4 ; yeast extract, $\left.2 ; \mathrm{NaCl}, 2\right)$ solidified with $8 \mathrm{~g}$ Gel-Gro ${ }^{-1}$ (ICN) for greater optical clarity. Tetrazolium red (2,3,5-tetraphenyltetrazolium chloride, TTC) was used at a concentration of $0.05 \%$.

Subsurface twitching assay. The $P$. aeruginosa colony to be tested was stab-inoculated through the agar to the underlying Petri dish. After incubation at 30 or $37^{\circ} \mathrm{C}$ for the specified times, the zone of motility at the agar/Petri dish interface was measured. To aid visualization, either the vital dye TTC was added to the medium prior to plate pouring, or the agar was compressed after incubation and stained using a $0.05 \%$ Coomassie brilliant blue R250 stain (40\% methanol, 10\% acetic acid) as described previously (Alm \& Mattick, 1995).

Light microscopy. Sterile microscope slides were submerged in molten Gel-Gro medium to obtain a thin layer of medium coating the slide. The slides were allowed to set in a horizontal position and air-dried briefly prior to use. The slides were then inoculated with a small loop-full of bacteria taken from an overnight plate culture. A sterile glass coverslip was placed over the point of inoculation and the slide transferred to a large Petri dish containing a moist tissue and sealed with Nescofilm (BANDO) to maintain humid conditions. Incubation times ranged from 2 to $6 \mathrm{~h}$ at $37^{\circ} \mathrm{C}$.

Slide cultures were examined using a Zeiss microscope Axioskop 50 with Nomarski facilities at 200-400× magnification. Video microscopy was performed in a room heated to $30^{\circ} \mathrm{C}$. Video images were recorded over a period of $2-4 \mathrm{~h}$ 
at speeds of either 1 field per $3.22 \mathrm{~s}, 1$ field per $0.66 \mathrm{~s}$ or real time (1 field per $1 / 50 \mathrm{~s}$ ) using a JVC TK-CI38IEG video camera connected to a Sanyo TLS-S2500P time-lapse video recorder. Video images were edited and converted to Quicktime movies using Avid Videoshop version 3.0 and can be viewed at http://www.cmcb.uq.edu.au/cmcb/PUBS/ twitch.html

Western analysis. Colony Western analysis was performed using the method described by McMichael (1992). An LB agar plate was stab-inoculated and incubated overnight at $37^{\circ} \mathrm{C}$. Following inoculation the agar was divided into quarters and one quarter was removed from the Petri dish, inverted and placed onto Hybond-C nitrocellulose membrane (Amersham). Several layers of blotting paper were placed on top of the membrane with a small weight. After $10 \mathrm{~min}$, the nitrocellulose was removed and rinsed in PBS. The membrane was blocked in $5 \%$ skim milk powder in PBS overnight and then exposed to a 1:1000 dilution of rabbit anti-PilA antibody in $1 \%$ skim milk powder in PBS. This was followed by exposure to a 1:10000 dilution of goat anti-rabbit immunoglobulin $G$ conjugated with horseradish peroxidase in $1 \%$ skim milk powder in PBS. The proteins were detected using the Supersignal chemiluminescence kit (Pierce) and exposed to X-ray film.

\section{RESULTS}

\section{Colony expansion due to twitching motility}

Fig. 1. shows the appearance of colonies of wild-type and a mutant of the fimbrial subunit gene ( $p i l A)$ of $P$. aeruginosa on the surface of an agar plate cultured in a humid environment, as well as at the interstitial surface between the agar and the plastic at the bottom of the plate. Wild-type colonies on the surface form flat, spreading colonies with a characteristic 'rough' appearance and a small twitching zone consisting of a very thin layer of cells (Fig. 1a). In contrast strains which are incapable of twitching motility (e.g. PAKpilA:: $\mathrm{Tc}^{\mathrm{R}}$ ) produce smooth domed colonies (Fig. 1b).

When wild-type $P$. aeruginosa cells are stabbed through an agar layer to the underlying plastic of the Petri dish, colony expansion due to twitching motility occurs very rapidly and is seen as a faint halo at the interstitial surface between the agar and the plastic (Fig. 1c). This does not occur in the PAKpilA:: $\mathrm{Tc}^{\mathrm{R}}$ strain (Fig. 1d) or in any mutants lacking twitching motility (Darzins, 1993; Alm \& Mattick, 1997). This halo consists of essentially a single layer of cells moving out from the colony and is much more easily visualized by staining with Coomassie blue or the vital dye TTC (Fig. 2a, b). TTC has been used by others to examine swimming motility in E. coli and gliding motility in M. xanthus (Berg \& Turner, 1995; Shi et al., 1996). TTC is a clear compound which is reduced to form a large insoluble red formazan crystal when taken up by the cell and has the advantage that, unlike Coomassie blue, it can be used to stain cells in situ.

Both Coomassie blue and TTC staining reveal a series of concentric rings in the twitching zone. Microscopic examination of the TTC stained zones revealed that the darker regions corresponded to areas of closely clustered microcolonies, whereas the lighter regions are comprised of a uniform layer of cells (data not shown). These microcolonies are reminiscent of those observed in the development of biofilms on plastic, which are also dependent on the presence of functional type IV fimbriae (O'Toole \& Kolter, 1998). The appearance in the $P$. aeruginosa colony expansion zone of regions of interspersed high and low cell densities suggests that colony expansion by twitching motility may involve some type of periodicity, which was confirmed by examining the kinetics of the process (see below). The denser TTCstained rings containing microcolonies do not appear until later in the incubation period, so we are unsure about how their position precisely relates to the periodicity of twitching motility.

We have calculated the overall rate of radial colony expansion at the interstitial surface between the LB agar and the plastic Petri dish to be about $0.6 \mathrm{~mm} \mathrm{~h}^{-1}$ by measuring the differences in the diameter of the colonies over a $26 \mathrm{~h}$ period with incubation at $37^{\circ} \mathrm{C}$. We also followed the kinetics of this expansion process more closely by measuring the diameter of the twitching zone every $30 \mathrm{~min}$ for a $12 \mathrm{~h}$ period with incubation at $30^{\circ} \mathrm{C}$ (Fig. 2c). These data suggest that twitching involves phases of motility interspersed with phases of cellular growth and division (consolidation), consistent with the periodicity implied by the patterns observed in Coomassie blue and TTC staining of the twitching zone (Fig. $2 \mathrm{a}, \mathrm{b})$.

To determine whether the fimbriae are expressed only in the twitching zone or across the colony as a whole, we performed a Western blot of a mature colony at the agar/plastic interface using an antibody which detects PilA (Fig. 2d, e). The results show that the fimbriae are expressed only in the outermost part of the zone, wherein twitching motility is actively taking place. This is also evidence of differentiation within the colony as a whole, indicating that both the production of fimbriae and presumably also the rate and direction of cellular motion, are being regulated by local environmental factors.

Colony expansion due to twitching motility at the surface of agar is significantly (about 10-fold) slower than that observed at the agar/plastic interface (cf. Fig. $1 \mathrm{a}$ and $\mathrm{c}$ ). To investigate if this might be due to differences in oxygen tension experienced in these environments we compared colony expansion both at the surface and beneath the agar under aerobic and microaerophilic conditions (the latter using a sealed chamber from which the oxygen had been reduced by burning a candle until extinguished), ensuring high humidity was maintained. We also assayed the interstitial twitching zone sizes at different volumes of agar $(5-40 \mathrm{ml})$. Neither approach demonstrated any significant effect on twitching motility (data not shown), indicating that differences in oxygen tension do not account for the observed rate differences. However, a halo of twitching motility identical to that observed at the interstitial surface between the agar and Petri dish 

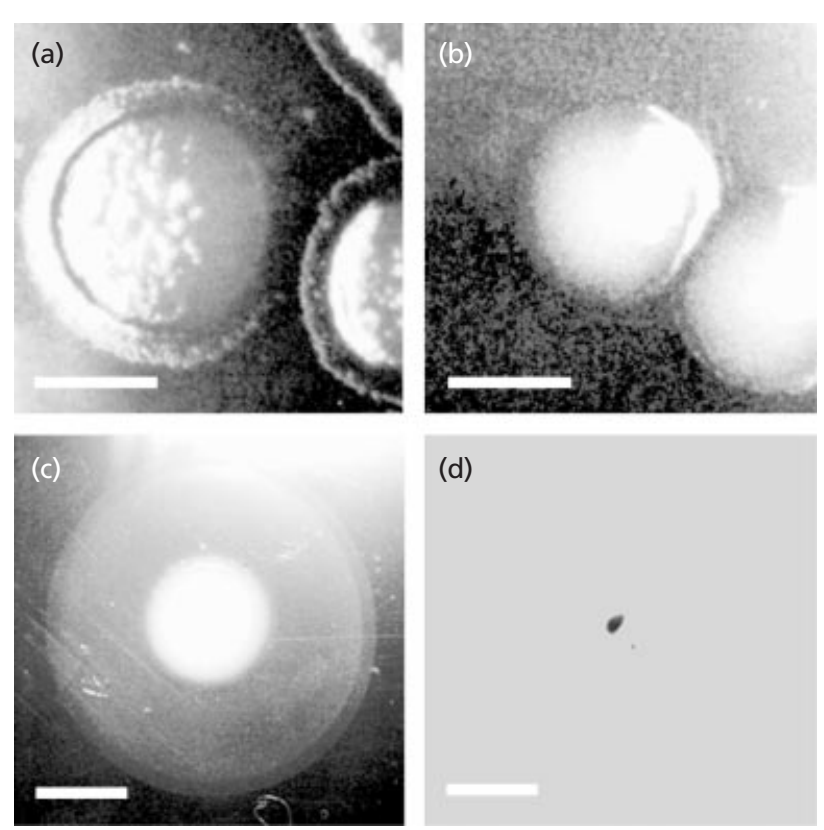

(d)

Fig. 1. Macroscopic views of $P$. aeruginosa colony morphologies after overnight incubation at $37^{\circ} \mathrm{C}$. (a) and (b) show surfacegrown colonies of PAK (wild-type) and PAKpilA::Tc ${ }^{R}$, respectively. Bars, $2 \mathrm{~mm}$. (c) and (d) show colonies obtained at the interstitial surface between the agar and Petri dish of PAK and PAKpilA:: $\mathrm{Tc}^{\mathrm{R}}$, respectively. Bars, $5 \mathrm{~mm}$. Note (d) has been stained with Coomassie blue to aid visualization of the colony.

can be formed on the surface of the agar by placing a glass coverslip over the top of cells which have been inoculated on an agar surface. This approach was used to study the micromorphological details of twitching motility by video microscopy (see below). We then examined whether twitching motility requires an abiotic surface or simply any solid surface above and below the cells (such as an agar-agar interface). We repeated the experiment with a thin agar slab in place of a glass coverslip and found that these conditions also promoted twitching motility, albeit not as actively as that observed at the agar-plastic interface at the bottom of a Petri dish. These observations suggest that twitching motility in $P$. aeruginosa is favoured at interstitial surfaces, including agar-plastic/glass or agar-agar surfaces.

However, to our surprise, during the course of these experiments we also found that active twitching motility occurred (at the same rate as at an agar-plastic interface) over the surface of agar that had been set against the plastic on the bottom of the plate and then inverted and exposed to the air (i.e. without an agar or glass cover). These twitching zones at the agar-air interface were identical to those observed at the agar-plastic interface, including the formation of concentric rings and the very fine outermost edge where twitching is actively taking place (see Figs 1c and 2a,b,d). This observation indicates that the requirements for twitching motility are not an interstitial surface per se but simply rather a smooth surface across which the bacteria can freely (a)

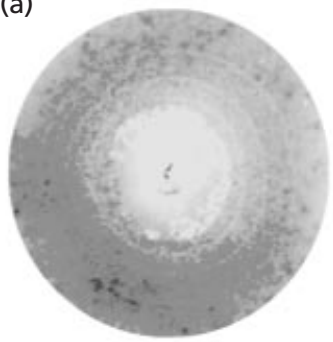

(b)

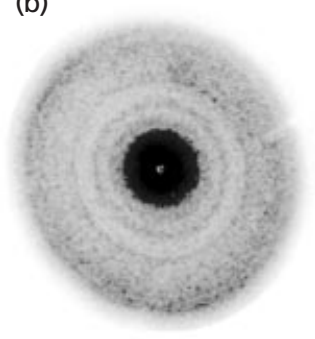

(c)

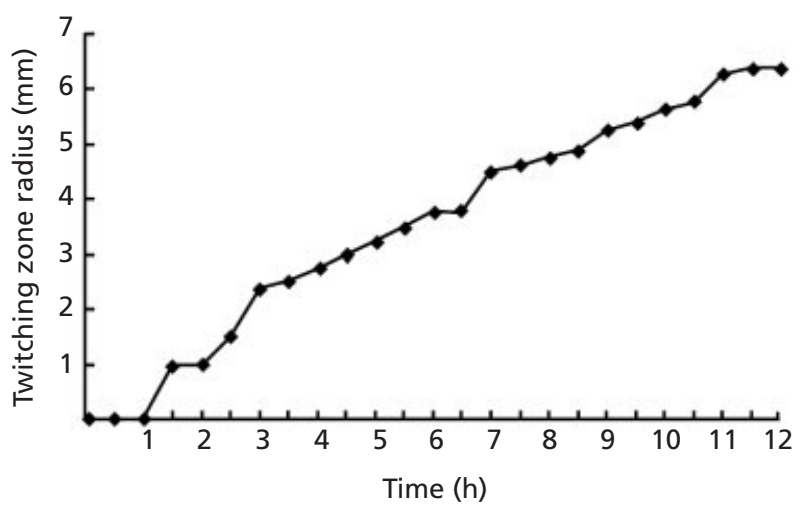

(d)

(e)

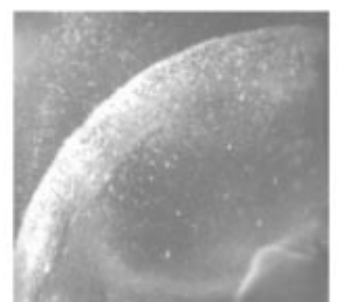

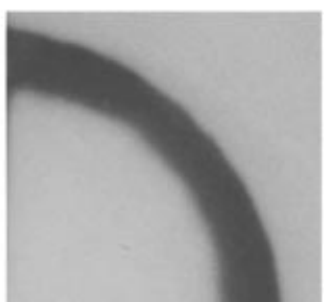

Fig. 2. Expansion profiles of twitching motility. (a) and (b) show interstitial twitching zones which have been either stained with Coomassie blue or grown in the presence of TTC, respectively. (c) is a graph of the radial distances of colony expansion measured at $30 \mathrm{~min}$ intervals over $12 \mathrm{~h}$. (d) and (e) show a whole-colony Western blot. (d) shows the macroscopic twitching zone of a PAK colony obtained at the interstitial surface of the agar and Petri dish. (e) is an autoradiograph of the same zone in (d) probed with anti-PilA antisera demonstrating that only the outermost periphery of the zone is expressing type IV fimbriae.

move. Presumably the very limited twitching motility normally observed on the surface of agar plates relates to the uneven or rough surface produced during the setting of the agar at the air interface, whereas the agar surface formed on the bottom against the plastic is very even. These observations indicate that active twitching motility can occur both at interstitial surfaces and on (smooth) biotic or abiotic surfaces exposed to air or liquid, consistent with the role of twitching motility in biofilm formation and what might be expected to occur during infection of epithelial surfaces. The difficulty in obtaining active twitching motility on the dried surface of conventional plates may also apply to other bacteria. 

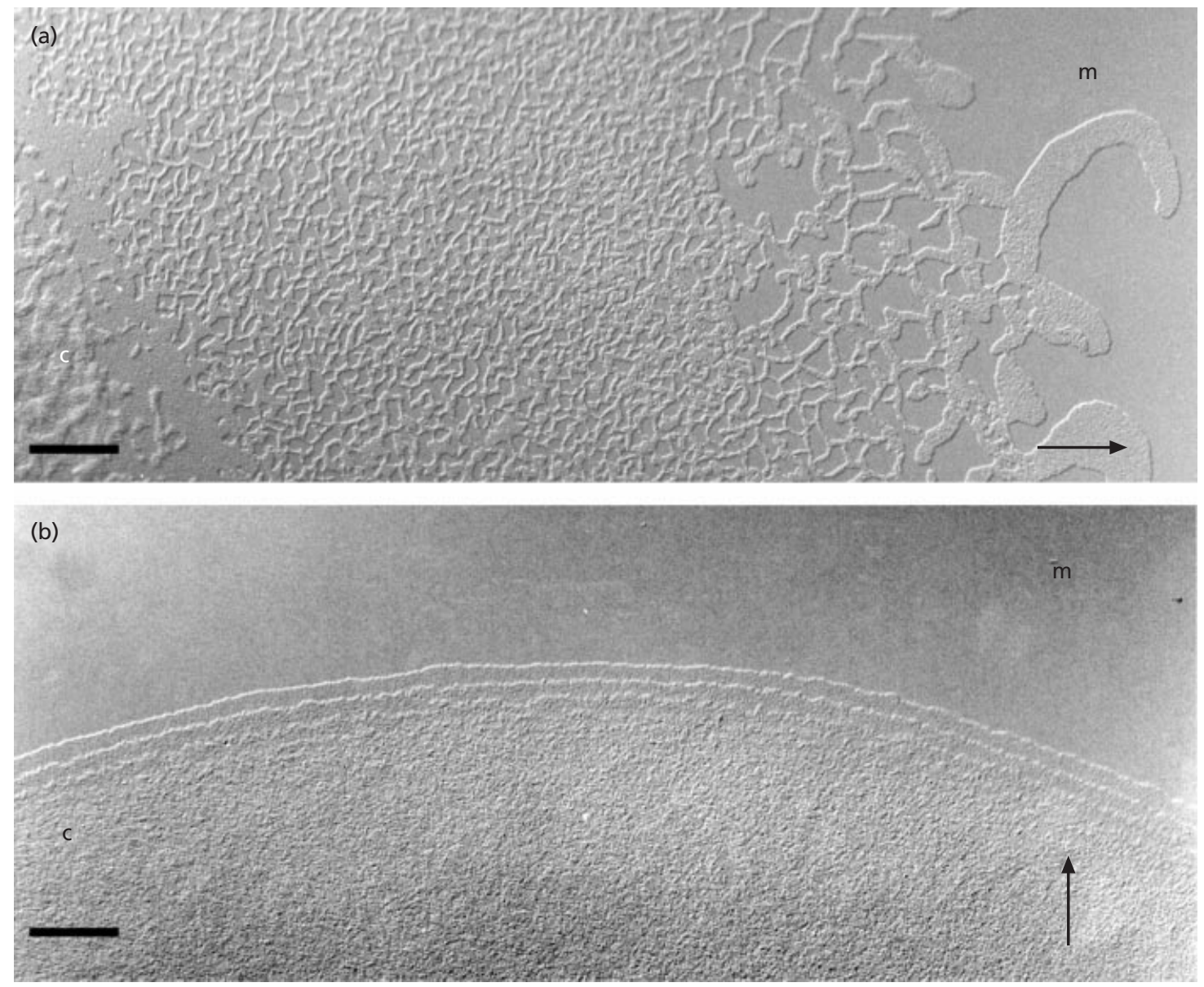

Fig. 3. Light microscopy of zones of twitching motility. (a) and (b) show typical colony expansion zones obtained at the interstitial surface between the glass coverslip and Gel-Gro medium for PAK (twitching) and PAKpilA::Tc ${ }^{R}$ (nontwitching) respectively. Micrographs were taken after $4-6 \mathrm{~h}$ incubation at $37^{\circ} \mathrm{C}$. Bars, $50 \mu \mathrm{m}$. ' $\mathrm{c}$ ' indicates the colony; ' $\mathrm{m}$ ' indicates the uncolonized medium; the arrow indicates the radial direction of colony expansion.

On this basis we suggest that the use of smooth agar surfaces (inverted after setting) may also allow twitching motility to be observed more easily in other type IV fimbriate species, such as $N$. gonorrhoeae and $N$. meningitidis, where thus far it has been very difficult to observe this process in vitro. We are currently examining this possibility.

\section{Microscopic analysis of the twitching zone}

We have developed a system for light microscopic analysis of twitching motility by adapting the method originally developed for this purpose by Darzins (1993), wherein cells were point-inoculated onto an agar block and covered by a glass slide, by replacing the agar with a thin layer of gellan gum (Gel-Gro) that gives much greater optical clarity. Under these conditions twitching motility is active and produces zones comparable to that observed on LB agar at the agar-plastic interface, as discussed above. Fig. 3(a) shows a typical micrograph of a twitching zone under glass, in comparison with that observed with a pilA mutant (Fig. 3b). The micro- morphological pattern of the twitching zone under glass differs substantially from that observed at the outermost edge of a surface-grown colony where, historically, twitching motility has been examined (see Bradley, 1980; Henrichsen, 1972, 1983), but which we now know to be a suboptimal environment for the process.

The twitching motility zone under optimal conditions is comprised of three major micromorphological patterns (Fig. 3a). The leading edge of the twitching zone exhibits large rafts of $10-50$ cells moving away from the colony edge. These motile rafts are characteristic of twitching motility and have been described before (Darzins, 1993; Bradley, 1980; Henrichsen, 1972). However, our results reveal that twitching motility can display far greater complexity than has been previously described. Behind these rafts a strikingly intricate lattice-work pattern is formed comprised of structures consisting of only $1-5$ cells in width. Closer to the colony, the spaces between the lattice are filled in, presumably due to cell division. It is also worth noting that the twitching zone is formed essentially of a single layer of cells. This is not 

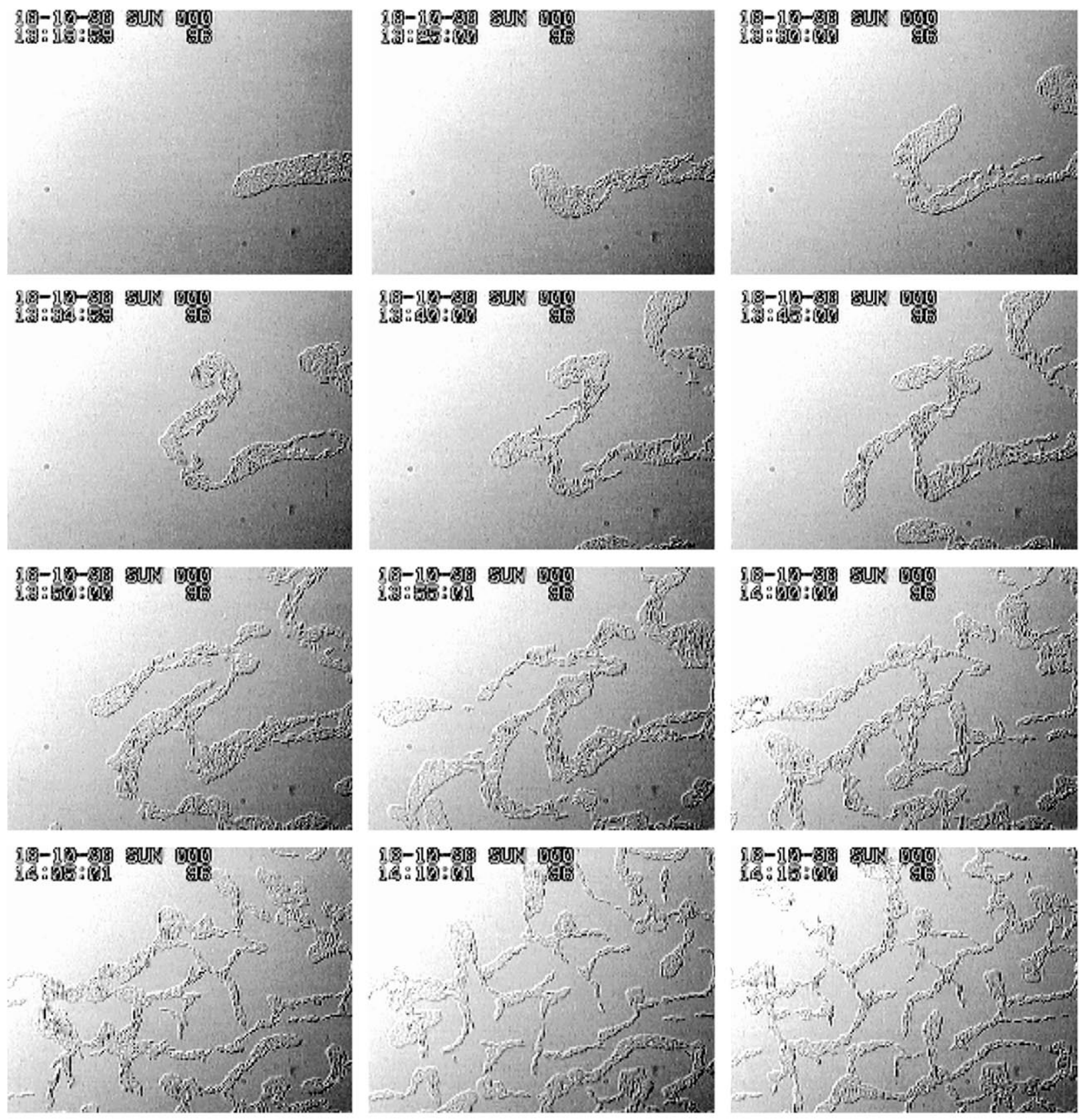

Fig. 4. PAK twitching motility filmed using time-lapse video microscopy. Captured frames shown here cover a $1 \mathrm{~h}$ period of real time. A Quicktime video of this sequence (and others) can be found at http://www.cmcb.uq.edu.au/cmcb/ PUBS/twitch.html

due to physical constraints as the centre of the colony itself and the microcolonies within the twitching zone are clearly capable of forming three-dimensional cell aggregates at the surface between agar or Gel-Gro and plastic or glass surfaces. Mutants which lack fimbriae do not exhibit raft or lattice formation (Fig. 3b). On a conventional agar surface (without a glass coverslip) (Fig. 1a) (see also Bradley, 1980; Henrichsen, 1972, 1983), wild-type colonies do exhibit aggregates of cells protruding from the colony edge, but lack the highly structured lattice-work observed under conditions (smooth surfaces) which promote active twitching motility (see above).

\section{Time-lapse video microscopy}

Time-lapse video microscopy confirmed that the initial stages of twitching motility involve the formation of large rafts of aggregated cells moving away from the colony edge (Fig. 4). These rafts move unidirectionally 

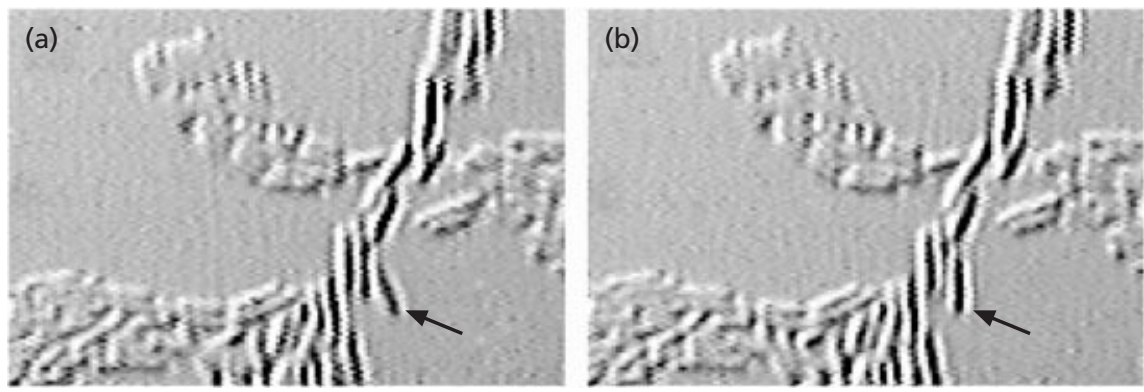

Fig. 5. Twitching motility. This sequence demonstrates the twitching action often observed when a cell snaps into alignment with neighbouring cells. The arrow indicates a cell approaching a group of cells end on until it touches another cell with its pole (a) at which point it snaps rapidly into alignment (b). This motility was filmed in real time and consecutive frames are shown.

following the long axis of the cells which are in tight cell-cell contact and generally move radially outward, although they do meander. Occasionally within these rafts individual cells can be seen to reverse direction and move back towards the colony, against the overall forward motion of the raft. Behind the rafts groups of cells appear to stretch out and break up into smaller aggregates which move off in different directions and connect with other groups (Fig. 4). This ultimately leads to the formation of the fine lattice-like network observed in the twitching zone (Figs $3 a$ and 4). The leading aggregates appear to lay down 'trails', along which following cells move rapidly up and down as individual units or small groups, often reversing direction but always following the long axis of the cell (Fig. 4). These 'trails' are not visible but their presence is inferred by the observation that following cells tend to follow pathways that have been previously traversed by other cells. Once the lattice has been formed, the bacteria within mature sections appear to settle and divide to fill out the colonized space (Fig. 3a).

The movement of the cells within the twitching zone is interesting. Individual cells appear to align with others along their long axis, apparently in intimate contact, and to slide by each other, either singly or in small groups. This is particularly noticeable and most active during the lattice formation, whereas the larger groups of cells in the leading rafts tend to move more in unison, although individual cells do appear to move around within the raft. Nonetheless, individual cells moving within the trails/lattice network move more rapidly than those in the rafts. In addition, reversal of movement also occurs more often in these trails than in the rafts.

Careful examination of numerous time-lapse recordings of twitching motility indicated that the movement involves cell-cell contact. Under the conditions used here, a single cell was never observed moving when well separated from other cells. Occasionally, an individual cell will move from one cluster to another but always when the clusters are in close proximity. Such a cell at first moves end on towards other cells until it touches another cell with its pole. It then immediately snaps into an adjacent position alongside the other cell (Fig. 5). This 'snapping' behaviour is very rapid and accounts for the jerky 'twitching' which is characteristically seen when this motility is observed without time-lapse (Bradley, 1980; Henrichsen, 1972, 1983). Individual cells are also seen frequently to move quickly along the outside of an existing trail of cells. The cells in the network appear to be in a generally constant state of movement, but individual cells can be stationary for a period and then suddenly move. All movement involves at least one cell contacting another with its pole, consistent with the polar location of the fimbriae. The impression is that the cells are pulling and pushing against each other.

We have calculated the rates of cell movement during twitching motility by analysis of our time-lapse video recordings. Under these conditions (Gel-Gro nutrient medium at $30^{\circ} \mathrm{C}$ ) the leading edge rafts can move at speeds of up to $6 \mu \mathrm{m} \mathrm{min}{ }^{-1}$, but can also temporarily stop, and normally move at a rate of about $3-4 \mu \mathrm{m}$ $\min ^{-1}$, consistent with macroscopic measurements of the radial expansion of the colony as a whole under these same conditions, which was measured to be $0 \cdot 16 \mathrm{~mm} \mathrm{~h}^{-1}$ over $16 \mathrm{~h}$ (about $2.6 \mu \mathrm{m} \mathrm{min}^{-1}$ - see also below). The rate of colony expansion due to cell division in the absence of twitching motility (calculated from analysis of time-lapse recordings of the pilA mutant) was only $0.1 \mu \mathrm{m} \mathrm{min}{ }^{-1}$, i.e. only a few percent of that mediated by twitching motility in the wild-type. Within the developing lattice behind the outgoing rafts, individual cells appeared to be more active and were measured to move up and down the trails at speeds of about $5-10 \mu \mathrm{m} \mathrm{min}{ }^{-1}$.

We have also observed that the rate of twitching motility is dependent on growth conditions. Using LB agar we have observed twitching-motility-mediated radial colony expansion rates of around $0.5-0.6 \mathrm{~mm} \mathrm{~h}^{-1}(8-10 \mu \mathrm{m}$ $\min ^{-1}$ ) at $30-37^{\circ} \mathrm{C}$, which appears to be a consequence of the higher nutrient levels in this agar than of the GelGro nutrient medium used for microscopic studies. Twitching motility is stimulated (further) by higher levels of tryptone and yeast extract, but not glucose (data not shown), and we are currently attempting to identify the particular components which are responsible for this stimulation.

\section{DISCUSSION}

Our results indicate that under appropriate conditions twitching motility in $P$. aeruginosa manifests as a rapid, highly organized mechanism of bacterial solid surface translocation. This description differs significantly from 
the picture presented by earlier studies of twitching motility which essentially stated that twitching motility is a relatively slow, disorganized mode of translocation in which cells move predominantly singly in an intermittent and jerky fashion (Henrichsen, 1972). Notably, these and other early studies examined twitching motility at the outer edge of the colony grown on an agar surface solidified and dried in air, which is clearly suboptimal for observing this process in vitro.

Conditions which appear to promote twitching motility in $P$. aeruginosa are growth of the cells on smooth solid or semi-solid surfaces, such as agar set against plastic. Type IV fimbriae are expressed only in the outer active twitching zone of expanding colonies (Fig. 2d, e), implying that type IV fimbriae are involved in both adhesion to and movement across such surfaces, consistent with the known properties of these organelles. Type IV fimbriae and twitching motility have also recently been shown to be required for biofilm formation by $P$. aeruginosa on abiotic surfaces (O’Toole $\&$ Kolter, 1998) and to be associated with the formation of microcolonies on these surfaces. Our observation of microcolonies within the twitching motility zone on agar culture is consistent with this and suggests that we are observing the rapid formation by twitching motility of a biofilm on the agar or Petri dish surface. Biofilm formation is also important in $P$. aeruginosa infections particularly in the lungs of cystic fibrosis patients where this pathogen grows as microcolonies embedded in the exopolysaccharide alginate (Govan \& Deretic, 1996). Twitching motility and alginate production have been shown to be coordinately (and probably reciprocally) regulated through AlgR (Whitchurch et al., 1996). Twitching motility therefore appears to be a key component of biofilm formation on both abiotic and biotic surfaces. In support of this, there is evidence to suggest that twitching motility plays a significant role in the colonization of epithelial surfaces in animals and the subsequent spread of infection as mutants which retain type IV fimbriae, but have lost twitching motility, have reduced infectivity (Hazlett et al., 1991; Comolli et al., 1999).

Our observations indicate that twitching motility shares many points of similarity with another mechanism of bacterial translocation known as gliding motility and is almost certainly synonymous with it. In Henrichsen's 1972 review, in which he surveyed and attempted to classify the various forms of bacterial surface translocation that had been reported in the literature up until that time, he states (in reference to gliding motility in a range of bacteria, notably fruiting and non-fruiting myxobacteria, such as Myxococcus and Cytophaga spp.) that, "Under conditions optimal for gliding, the colonies will be seen as "completely flat, rapidly spreading almost invisible swarms" or as a spreading, rhizoid growth with a honeycomb appearance. Movement takes place mainly in "spearheads" (i.e. spearhead-shaped cell aggregates at the edge of the colony), single isolated cells very rarely being motile, and the picture is one of a "changing dispersed border" with interlacing bands being continuously rearranged.' A similar description is given elsewhere in the same review: 'The cells are arranged in a loose pattern of interlacing bands of rafts and cells... Groups of cells resembling spearheads are seen projecting outwards. The locomotion, which is principally seen in the groups of cells, i.e. rafts and spearheads, and takes the direction of the longitudinal axis of the bacteria, gives rise to a constantly changing picture, steadily gliding groups of cells uniting or dividing...' (Henrichsen, 1972).

This is an exact description of what we have observed by video microscopy as type IV fimbriae-dependent twitching motility in $P$. aeruginosa at the agar/Gel-Groplastic/glass interface. Henrichsen also reported that gliding motility is confined to solid surfaces such as glass and agar, requires high humidity and that at least some species which exhibit gliding motility possess surface filaments of 4-8 nm, all of which are characteristic of twitching motility or type IV fimbriae. It therefore appears that Henrichsen's conclusion that twitching and gliding motility are distinctive processes reflects artefactual differences observed in vitro in different species due to suboptimal environmental conditions, as well as perhaps in some cases species-specific idiosyncrasies in the process or its regulation and directional control. In fact, prior to Henrichsen's review there had been some debate as to whether or not twitching motility and gliding motility were the same process (see Henrichsen, 1972 and references therein).

The conclusion that twitching and gliding motility are alternative names for essentially the same process is also supported by microscopic and molecular genetic studies of gliding motility in M. xanthus, wherein the process has been extensively studied over the past decade. Gliding motility in this species has been separated into two facets, namely 'adventurous motility', involving the movement of single cells away from the colony by an unknown mechanism, and 'social motility', which has many micromorphological similarities with our observations of twitching motility. Both twitching and social gliding motility appear to involve trail formation. In both cases, cells moving within large rafts do not reverse as frequently as cells moving in trails (Shi et al., 1996). Our observation that in twitching motility isolated cells are incapable of motility and that translocation only occurs when cells are in close proximity suggests that cell-cell interactions are required for motility, which is consistent with observations of social motility in $M$. xanthus $\mathrm{A}^{-} \mathrm{S}^{+}$mutants (Hodgkin \& Kaiser, 1979).

Furthermore, social gliding motility in M. xanthus has been recently shown to be dependent on type IV fimbriae, based on the discovery that several genes required for this process (pilA, pilS, pilR, pilB, pilC, pilD, pilT and pilQ) (Wu \& Kaiser, 1995, 1997; Wu et al., 1997; Wall et al., 1999) are homologous to genes previously identified in $P$. aeruginosa as being required for fimbrial biogenesis and twitching motility (Alm \& Mattick, 1997), consistent with earlier electron microscopic studies that indicated that pili or fimbriae were 
important in social motility (Kaiser, 1979). At the same time, studies of twitching-impaired mutants of $P$. aeruginosa showed that this process was dependent on a set of genes ( $p i l G H I J K L, c h p A B$ ) encoding a chemotactic signal transduction system (Darzins, 1993, 1994, 1995; Alm \& Mattick, 1997), similar to that involved in controlling flagellar-mediated swimming in enteric bacteria, but which is more complex and most closely related to an equivalent set of genes (frzABCDEFG) controlling social gliding motility in $M$. xanthus (McBride et al., 1989; McCleary \& Zusman, 1990).

The mechanism of twitching/gliding motility remains a mystery. The comparative molecular genetics, genomics and biochemistry of twitching/gliding motility in $P$. aeruginosa and $M$. xanthus adds a powerful new dimension to dissecting this process. Clearly, in both cases type IV fimbriae are involved, as is cell-cell contact involving at least one pole, where the fimbriae are located. In $P$. aeruginosa, in most cases (except when cells intersect at an angle), the cells are aligned along their long axis and appear to slide by each other. Cell alignment also appears to be involved in M. xanthus gliding motility and to be important in a process termed 'stimulation' whereby M. xanthus $\operatorname{tgl}^{-}$mutants which lack type IV fimbriae and gliding motility can be rescued phenotypically in both respects by physical contact with tgl ${ }^{+}$cells (Rodriguez-Soto \& Kaiser, 1997; Wall et al., 1998; Wall \& Kaiser, 1998). Importantly, this stimulation involves Tgl-mediated stimulation of fimbrial assembly from pre-existing pilin and to lead to a rate of fimbrial elongation in $\mathrm{tgl}^{-}$mutants that is similar to wild-type, which is broadly consistent with the conclusion from electron microscopic studies of $P$. aeruginosa that type IV fimbriae can extend and retract (Bradley, 1972a, b, 1974). Comparison of proteins known to be involved in twitching motility in $P$. aeruginosa to $\mathrm{Tgl}$ indicates that PilF is significantly similar to $\mathrm{Tgl}$ across the length of the protein and thus may serve an equivalent function in twitching motility as a trigger for contact-initiated fimbrial assembly/ extension/retraction in this organism (Watson et al., 1996a; J. S. Mattick \& C. B. Whitchurch, unpublished observations). This hypothesis is currently being tested.

The helical structure of the fimbrial strand strongly implies that the motive force for twitching/gliding motility is transduced by fimbrial rotation, either as part of the extension/retraction of the fimbrial strand per se (Mattick \& Alm, 1995) or by rotational forces applied externally to the cell or adjacent cells. In this context it is interesting to note that electron microscopic examination of cells within the colony of the twitching bacterium $N$. gonorrhoeae has demonstrated that the type IV fimbriae of this bacterium wrap around the cells from which they emanate as thin, individual structures, but also form thick bundles which branch and subdivide to interconnect cells within the colony (Todd et al., 1984). It is our impression that the cells may be swivelling during twitching in $P$. aeruginosa, but the micrographic resolution is insufficient to be confident about this. Whether twitching/gliding motility is induced by the fimbriae acting in cis on their home cell or in trans by pushing or pulling adjacent cells remains to be determined. In relation to the latter, our video microscopy of $P$. aeruginosa, especially of isolated cells that are being reconnected to existing trails, would suggest that the cells are, if anything, being pulled rather than pushed.

Despite semantic differences and varying use of nomenclature, there now appears to be three systems of bacterial motility that are genetically and morphologically well-defined: (1) swimming motility, which occurs in fluid media and is mediated by reversible rotation of flagella; (2) swarming motility, which occurs on solid surfaces and is mediated by elongated and peritrichously hyperflagellated swarmer cells; and (3) twitching motility/social gliding motility which also occurs on solid surfaces and is mediated by type IV fimbriae. Interestingly, we have recently found that $P$. aeruginosa is also capable of another form of motility which resembles swarming, and that this activity can occur in concert with twitching motility, although it is distinct from it (C. B. Whitchurch \& J. S. Mattick, unpublished observations). Thus $P$. aeruginosa appears to have the capacity for all three of these forms of motility, under different circumstances, further evidence of the versatility of this organism.

\section{ACKNOWLEDGEMENTS}

Annalese B. T. Semmler and Cynthia B. Whitchurch contributed equally to this work. This work was supported by grants to C.B.W. and J.S.M. by the National Health and Medical Research Council of Australia. The Centre for Molecular and Cellular Biology is a Special Research Centre of the Australian Research Council.

\section{REFERENCES}

Alm, R. A. \& Mattick, J. S. (1995). Identification of a gene, pilV, required for type 4 fimbrial biogenesis in Pseudomonas aeruginosa whose product possesses a prepilin-like leader sequence. Mol Microbiol 16, 485-496.

Alm, R. A. \& Mattick, J. S. (1997). Genes involved in the biogenesis and function of type-4 fimbriae in Pseudomonas aeruginosa. Gene 192, 89-98.

Barnett, T. C., Kirov, S. M., Strom, M. S. \& Sanderson, K. (1997). Aeromonas spp. possess at least two distinct type IV pilus families. Microb Pathog 23, 241-247.

Berg, H. C. \& Turner, L. (1995). Cells of Escherichia coli swim either end forward. Proc Natl Acad Sci USA 92, 477-479.

Bradley, D. E. (1972a). Evidence for the retraction of Pseudomonas aeruginosa RNA phage pili. Biochem Biophys Res Commun 47, 142-149.

Bradley, D. E. (1972b). Shortening of Pseudomonas aeruginosa pili after RNA-phage adsorption. J Gen Microbiol 72, 303-319.

Bradley, D. E. (1974). The adsorption of Pseudomonas aeruginosa pilus-dependent bacteriophages to a host mutant with nonretractile pili. Virology 58, 149-163.

Bradley, D. E. (1980). A function of Pseudomonas aeruginosa PAO pili: twitching motility. Can J Microbiol 26, 146-154.

Chi, E., Mehl, T., Nunn, D. \& Lory, S. (1991). Interaction of 
Pseudomonas aeruginosa with A549 pneumocyte cells. Infect Immun 59, 822-828.

Comolli, J. C., Hauser, A. R., Waite, L., Whitchurch, C. B., Mattick, J. S. \& Engel, J. N. (1999). Pseudomonas aeruginosa gene products PilT and PilU are required for cytotoxicity in vitro and virulence in a mouse model of acute pneumonia. Infect Immun 67, 3625-3630.

Dalrymple, B. \& Mattick, J. S. (1987). An analysis of the organization and evolution of type 4 fimbrial (MePhe) subunit proteins. J Mol Evol 25, 261-269.

Darzins, A. (1993). The pilG gene product, required for Pseudomonas aeruginosa pilus production and twitching motility, is homologous to the enteric, single-domain response regulator CheY. J Bacteriol 175, 5934-5944.

Darzins, A. (1994). Characterization of a Pseudomonas aeruginosa gene cluster involved in pilus biosynthesis and twitching motility: sequence similarity to the chemotaxis proteins of enterics and the gliding bacterium Myxococcus xanthus. Mol Microbiol 11, 137-153.

Darzins, A. (1995). The Pseudomonas aeruginosa pilK gene encodes a chemotactic methyltransferase (CheR) homologue that is translationally regulated. Mol Microbiol 15, 703-717.

Dorr, J., Hurek, T. \& Reinhold-Hurek, B. (1998). Type IV pili are involved in plant-microbe and fungus-microbe interactions. Mol Microbiol 30, 7-17.

Farinha, M. A., Conway, B. D., Glasier, L. M. G., Ellert, N. W., Irvin, R. T., Sherburne, R. \& Paranchych, W. (1994). Alteration of the pilin adhesin of Pseudomonas aeruginosa PAO results in normal pilus biogenesis but a loss of adherence to human pneumocyte cells and decreased virulence in mice. Infect Immun 62, 4118-4123.

Forest, K. T. \& Tainer, J. A. (1997). Type-4 pilus-structure: outside to inside and top to bottom - a minireview. Gene 192, 165-169.

Govan, J. \& Deretic, V. (1996). Microbial pathogenesis in cystic fibrosis: mucoid Pseudomonas aeruginosa and Burkholderia cepacia. Microbiol Rev 60, 539-574.

Hazlett, L. D., Moon, M. M., Singh, A., Berk, R. S. \& Rudner, X. L. (1991). Analysis of adhesion, piliation, protease production and ocular infectivity of several $P$. aeruginosa strains. Curr Eye Res 10, 351-362.

Henrichsen, J. (1972). Bacterial surface translocation: a survey and a classification. Bacteriol Rev 36, 478-503.

Henrichsen, J. (1975). The occurrence of twitching motility among Gram-negative bacteria. Acta Pathol Microbiol Scand Sect B 83, 171-178.

Henrichsen, J. (1983). Twitching motility. Annu Rev Microbiol 37, 81-93.

Henrichsen, J. \& Blom, J. (1975). Examination of fimbriation of some gram-negative rods with and without twitching and gliding motility. Acta Pathol Microbiol Scand Sect B 83, 161-170.

Hobbs, M. \& Mattick, J. S. (1993). Common components in the assembly of type 4 fimbriae, DNA transfer systems, filamentous phage and protein secretion apparatus; a general system for the formation of surface-associated protein complexes. Mol Microbiol 10, 233-243.

Hobbs, M., Collie, E. S. R., Free, P. D., Livingston, S. P. \& Mattick, J. S. (1993). PilS and PilR, a two-component transcriptional regulatory system controlling transcription of type 4 fimbriae in Pseudomonas aeruginosa. Mol Microbiol 7, 669-682.

Hodgkin, J. \& Kaiser, D. (1979). Genetics of gliding motility in Myxococcus xanthus (Myxobacterials): Two gene systems control movement. Mol Gen Genet 171, 177-191.
Kaiser, D. (1979). Social gliding is correlated with the presence of pili in Myxococcus xanthus. Proc Natl Acad Sci USA 76, 5952-5956.

Lautrop, H. (1961). Bacterium anitratum transferred to the genus Cytophaga. Int Bull Bacteriol Nomencl Taxon 11, 107-108.

Liles, M. R., Viswanathan, V. K. \& Cianciotto, N.P. (1998). Identification and temperature regulation of Legionella pneumophila genes involved in type IV pilus biogenesis and type II protein secretion. Infect Immun 66, 1776-1782.

McBride, M. J., Weinberg, R. A. \& Zusman, D. R. (1989). "Frizzy", aggregation genes of the gliding bacterium Myxococcus xanthus show sequence similarities to the chemotaxis genes of enteric bacteria. Proc Natl Acad Sci USA 86, 424-428.

McCleary, W. R. \& Zusman, D. R. (1990). FrzE of Myxococcus xanthus is homologous to both CheA and CheY of Salmonella typhimurium. Proc Natl Acad Sci USA 87, 5898-5902.

McMichael, J. C. (1992). Bacterial differentiation within Moraxella bovis colonies growing at the interface of the agar medium with the Petri dish. J Gen Microbiol 138, 2687-2695.

Mattick, J. S. \& Alm, R. A. (1995). Common architecture of type 4 fimbriae and complexes involved in macromolecular traffic. Trends Microbiol 3, 411-413.

Mattick, J. S., Bills, M. M., Anderson, B. J., Dalrymple, B., Mott, M. R. \& Egerton, J. R. (1987). Morphogenetic expression of Bacteroides nodosus fimbriae in Pseudomonas aeruginosa. J Bacteriol 169, 33-41.

Mattick, J. S., Hobbs, M., Cox, P. T. \& Dalrymple, B. P. (1993). Molecular biology of the fimbriae of Dichelobacter (prev. Bacteroides) nodosus. In Genetics and Molecular Biology of Anaerobic Bacteria, pp. 517-545. Edited by M. Sebald. New York: Springer.

O'Toole, G. A. \& Kolter, R. (1998). Flagellar and twitching motility are necessary for Pseudomonas aeruginosa biofilm development. Mol Microbiol 30, 295-304.

Ottow, J. C. G. (1975). Ecology, physiology and genetics of fimbriae and pili. Annu Rev Microbiol 29, 79-108.

Parge, H. E., Forest, K. T., Hickey, M. J., Christensen, D. A., Getzoff, E. D. \& Tainer, J. A. (1995). Structure of the fibre-forming protein pilin at $2 \cdot 6 \AA$ resolution. Nature $378,32-38$.

Rodriguez-Soto, J.P. \& Kaiser, D. (1997). Identification and localization of the Tgl protein, which is required for Myxococcus xanthus social motility. J Bacteriol 179, 4372-4381.

Roine, E., Raineri, D. M., Romantschuk, M., Wilson, M. \& Nunn, D. N. (1998). Characterization of type IV pilus genes in Pseudomonas syringae pv. tomato DC3000. Mol Plant Microbe Interact 11, 1048-1056.

Rothbard, J. B., Fernandez, R., Wang, L., Teng, N. N. H. \& Schoolnik, G. K. (1985). Antibodies to peptides corresponding to a conserved sequence of gonococcal pilins block bacterial adhesion. Proc Natl Acad Sci USA 82, 915-919.

Ruehl, W. W., Marrs, C., Beard, M. K., Shokooki, V., Hinojoza, J. R., Banks, S., Bieber, D. \& Mattick, J. S. (1993). Q pili enhance the attachment of Moraxella bovis to bovine corneas in vitro. Mol Microbiol 7, 285-288.

Sambrook, J., Fritsch, E. F. \& Maniatis, T. (1989). Molecular Cloning: a Laboratory Manual, 2nd edn. Cold Spring Harbor, NY : Cold Spring Harbor Laboratory.

Shi, W., Ngok, F. K. \& Zusman, D. R. (1996). Cell density regulates cellular reversal frequency in Myxococcus xanthus. Proc Natl Acad Sci USA 93, 4142-4146.

Stone, B. J. \& Abu Kwaik, Y. (1998). Expression of multiple pili by 
Legionella pneumophila: identification and characterization of a type IV pilin gene and its role in adherence to mammalian and protozoan cells. Infect Immun 66, 1768-1775.

Todd, W. J., Wray, G. P. \& Hitchcock, P. J. (1984). Arrangement of pili in colonies of Neisseria gonorrhoeae. J Bacteriol 159, 312-320.

Wall, D. \& Kaiser, D. (1998). Alignment enhances the cell-to-cell transfer of pilus phenotype. Proc Natl Acad Sci USA 95, 3054-3058.

Wall, D., Wu, S. S. \& Kaiser, D. (1998). Contact stimulation of Tgl and type IV pili in Myxococcus xanthus. J Bacteriol 180, 759-761.

Wall, D., Kolenbrander, P. E. \& Kaiser, D. (1999). The Myxococcus xanthus pilQ ( $s g l A)$ gene encodes a secretin homolog required for type IV pilus biogenesis, social motility, and development. $J$ Bacteriol 181, 24-33.

Watson, A. A., Alm, R. A. \& Mattick, J. S. (1996a). Identification of a gene, pilF, that is required for type 4 fimbrial biogenesis and twitching motility in Pseudomonas aeruginosa. Gene 180, 49-56.
Watson, A. A., Mattick, J. S. \& Alm, R. A. (1996b). Functional expression of heterologous type 4 fimbriae in Pseudomonas aeruginosa. Gene 175, 143-150.

Whitchurch, C. B., Alm, R. A. \& Mattick, J. S. (1996). Co-ordinate regulation of fimbrial function and alginate production in Pseudomonas aeruginosa. Proc Natl Acad Sci USA 93, 9839-9843.

Wu, S. S. \& Kaiser, D. (1995). Genetic and functional evidence that type IV pili are required for social gliding motility in Myxococcus xanthus. Mol Microbiol 18, 547-558.

Wu, S. S. \& Kaiser, D. (1997). Regulation of expression of the pilA gene in Myxococcus xanthus. J Bacteriol 179, 7748-7758.

Wu, S. S., Wu, J. \& Kaiser, D. (1997). The Myxococcus xanthus pilT locus is required for social gliding motility although pili are still produced. Mol Microbiol 23, 109-121.

Received 12 March 1999; revised 4 June 1999; accepted 9 June 1999. 